\title{
Cyclogram and cross correlation: A comparative study to quantify gait coordination in mental state
}

\author{
Deepak Joshi ${ }^{1,2}$, Sneh Anand ${ }^{1,2}$ \\ ${ }^{1}$ Center for Biomedical Engineering, IIT Delhi, India; \\ ${ }^{2}$ Center for Biomedical Unit, AIIMS, New Delhi, India. \\ Email: joshideepak2004@yahoo.co.in
}

Received 19 December 2009; revised 28 December 2009; accepted 4 January 2010.

\begin{abstract}
The purpose of this study to evaluate the effect of mental task on gait coordination. The comparison between two techniques Crosscorrelation and Cyclogram has been performed. A set of gait experiments was developed and conducted to evaluate the effect of mental task on gait coordination. The perimeter derived from the geometric figure, cyclogram perimeter (CP), of the knee-knee cyclogram is the main descriptor considered in this study. For crosscorrelation it is the peak value of cross correlation coefficient (CCC) that has been taken for comparison. The sensitivity of both the techniques in terms of percentage has been calculated. Crosscorrelation is highly sensitive (mean=20.4 S.D.=2.3), towards the change in gait coordination with mental task, in comparison to cyclogram perimeter (mean=2.2 S.D.=1.2). The results have strength to assess the progress of rehabilitation among Parkinson patients.
\end{abstract}

Keywords: Gait Coordination; Cyclogram; Crosscorrelation; Mental Task

\section{INTRODUCTION}

It is no longer sufficient to consider gait as a mostly automatic motor activity, but rather one that requires the integration of motor function with cognitive processes such as attention, memory and planning. The evidence of improvement in gait disturbance in Parkinson's disease patients after giving training in mental singing also supports this concept. A focus on high level gait performance therefore requires a focus on cognition [1,2].

\subsection{Dual Task Interference}

Walking, Running, Swimming and other similar locomotive activities involve gross motor skills and the coordination to perform this is gross motor coordination. Gross motor coordination is left-right coordination, which re- fers to the alternating left and right limb movement. The basic neuronal circuits that generate this type of coordinated activity are located in the spinal cord. Similar to the lower limb there is left-right coordination in upper limb as well e.g., playing piano, drumming. In walking humans, arm to leg coordination is a well established phenomenon. It could be derived from the intrinsic organization of the human CNS (central Nervous System), but it could also consist of a movement induced epiphenomenon [3]. Arm and hand movements are mainly controlled by the motor cortical regions, whereas locomotion is thought to be regulated mainly at brain-stem, spinal, and cerebellar regions, with descending input from the cortex. Gait consists of highly preprogrammed movements, whereas some upper-extremity movements are more novel and thought to require attention, visual guidance, and somatosensory feedback to control their performance [4]. Interference between cognitive tasks and motor control activities such as gait is a problem in neurological rehabilitation settings. Interference between cognition and locomotor tasks may be important in assessing a neurological patients' ability to function independently, and in designing therapies for both cognitive and motor rehabilitation.

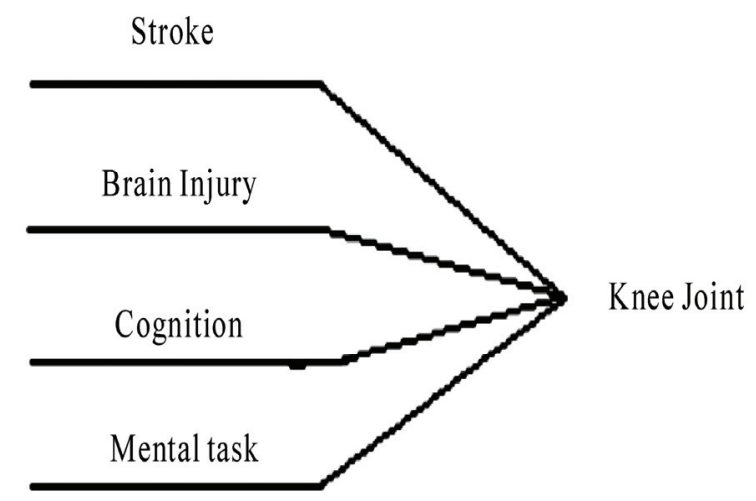

Figure 1. Cognitive aspects that affect knee joint motion. 
Quantification of the extent of interference between gait and cognitive tasks after brain injury has been reported. The walking speed of Alzheimer's disease patients slowed more than that of elderly subjects during the dual task. This may contribute to the risk of falls [5, 6]. People with chronic stroke incidence cope well with the challenges of varied environments and can maintain their gait speed while performing a secondary task. Despite moderate levels of gait impairment, gait automaticity may be restored over time to a functional level [7]. So many gait parameters has been studied for assessment of dual task interference. A recent research shows the gait parameters velocity, step time, swing time, and stance time have a significant interaction between complexity of mental task and articulation, with articulation having a greater effect at higher levels of complexity [8]. Very few researchers have taken the kinematic variables for the assessment of same. Knee joint has its leading role in most of the locomotion like walking, running squatting and sitting crossed leg (SCL). So knee angle can be the most affected parameter while monitoring dual task assessment. Figure 1 shows the knee joint cognition aspects. Time histories of neuromuscular and mechanical variables of human motion are often compared by using discrete timing events (onset, offset, time to peak, zero crossing, etc.). The determination of these discrete timing points is often subjective and their interpretation can cause confusion when attempting to compare patterns [9].

\subsection{Crosscorrelation}

Keeping in mind the matching of pattern of knee angle trajectories for various locomotion tasks has been monitored to assess the effect on mental task on locomotion activity. Crosscorrelation is a well-known and elegant method of detecting common periodicities between two signals of interest. Crosscorrelation is a measure of similarity of two waveforms as a function of a time-lag applied to one of them. For discrete functions, the crosscorrelation is defined as:

$$
(f * g)[\mathrm{n}]=\sum_{-\infty}^{+\infty} f[m] * g[n+m]
$$

where $f$ and $g$ are two time series, having $m$ samples each. The $n$ variable corresponds to the lag (in number of samples) by which the time series is shifted. The crosscorrelation gives an indication of pattern similarity between the two sets of data. In that sense Crosscorrelation is an objective means of pattern recognition and comparison. Crosscorrelation has been used to quantify coordination between joints of the same and different limbs during spontaneous kicking in 7 to 8 week old infants [10]. Study has been conducted to compare EMG signals from different walking trails, different test sessions, and different individuals in able-bodies adults. The results shows that crosscorrelation may be useful for evaluating changes in an individual patient's muscle activation patterns [11]. Another potential application of crosscorrelation was in monitoring the adaptations in interlimb and intralimb coordination to asymmetric loading in human walking. Changes in coordinative patterns were quantified utilization both crosscorrelation and root-meansquare difference (RMS) measures. Crosscorrelation measures were utilized to assess differences in the temporal evolution between coordination patterns [12].

\subsection{Cyclogram}

A cyclogram is formed by ignoring the time axis angle and directly plotting knee angle of one leg VS knee angle of other leg (Figure 3). Cyclograms reflect the gait kinematics during the total gait cycle which is different from having other discrete measures such as the step length, or walking speed, which are more common in literature. The geometrical features of cyclogram have been used to study the human walking in slope surface [13]. Simple iterative algorithm has been proposed for the computation of moments from a polygon approximation of the boundary, like in cyclogram. A discrete version of Green's theorem, which evaluates a double sum over a two dimensional discrete object by a simple summation along the discrete boundary of the object, was implemented [14,15]. The cyclograms are readily adaptable to clinical purposes by overlay of normal and abnormal gait traces. In human the pattern is speed dependent, highly predictable, and dramatically affected in the case of gait abnormalities. Comparison between bipedal and quadrupedal locomotion has been done by cyclogram [16]. Neural network approach was used to classify three gait patterns using the features of hip-knee cyclogram. Three gait patterns were generated from normal gait, a simulation of leg length difference, and a simulation of leg weight difference [17]. Cyclogram is also used for gait signature. If it is use as the signature in gait recognition and verification, it could lead to an automatic person recognition system using video footage from security cameras. To compare the signatures between two gaits, the difference of shape and phase of the cyclograms were calculated using the point projection method and extreme points of curves [18].Hip range, knee range, ratio of hip range/knee range, ratio of knee range/hip range, area of cyclogram, circularity, eccentricity, orientation, and cusp orientation [19]. No literature is available that reports use of cyclogram for assessing the dual task interference. Authors used the perimeter of knee-knee cyclogram to see the eefect of mental task on gait coordination. Further the results are compared with the previous work of authors in which the crosscorrelation between knee angles was used to assess the effect of mental task on gait coordination [20]. 
To compare the performance of both techniques the sensitivity of both, for gait with mental task is calculated as follows:

$\%$ sensitivity $(\mathrm{CC})=\{\mathrm{CCC}(\mathrm{NW})-\mathrm{CCC}(\mathrm{MT})\} / \mathrm{CCC}(\mathrm{NW})$

$\%$ sensitivity $(\mathrm{CP}))=\{\mathrm{CP}(\mathrm{NW})-\mathrm{CP}(\mathrm{MT})\} / \mathrm{CP}(\mathrm{NW})\}(3)$

Where CCC- peak of crosscorrelation coefficient, CPCyclogram Perimeter, NW-Normal Walk, MT-walking with mental task, CC-Crosscorrelation.

Besides Crosscorrelation and Cyclogram, Artifical Neural Network (ANN) can be most powerful tool to evaluate the coordination in different gait patterns [21,22]. Though ANN needs more computational power and is quite complex to be calculated in comparision of Crosscorrelation and Cyclogram, in real time. So authors compared the sensitivity of Crosscorrelation and cyclogram for this study.

\section{EXPERIMENTAL METHOD}

Six male healthy subjects (mean age $=26$ years, S.D. $=4.6 y e a r s$ ) without any history of lower extremity injury participated voluntarily for the experiment. All of them provided written consent to participate. Subjects were educated enough to carry out the mental task exercise. Data was collected in a $3 \mathrm{D}$ motion analysis system using six CCD Cameras. EVA 7.0 and Orhtotrak 6.2 software were used for data recording and gait analysis respectively. Twenty five Cleveland markers were placed on the subjects.

\subsection{Experiment Protocol}

Subjects were introduced to the various locomotive tasks initially, except walking with a mental task. This was done to avoid any biasing to the mental state while only walking. Data recording began 2-3 minutes after the subjects began walking. This was done to habituate the subjects to maintain constant speed. This speed was preferred speed. Seven to eight trials were performed for each locomotive task to get sufficient amount of data for comparison and analysis. After completing all locomotive tasks, they were asked to perform a mental task exercise along with walking. The mental task assigned to them was naming of months from December to January. Subjects were instructed not to skip any month in between and if they commit any mistake they should improve it. Thus difficulty level of the task was maintained.

\subsection{Data Collection}

Data was collected at the sampling frequency of $120 \mathrm{~Hz}$. The time duration to record the data for every trial was 3 seconds, except walking with mental task. Therefore the total sample for a trial was 360 . While having mental task the subjects was instructed to walk until they complete it. Some of the trials were not included for analysis as they were corrupted due to incomplete information or noise. A low pass Butterworth filter with cut off of 6.0 $\mathrm{Hz}$ was used to remove the noise from data. The Orthotrak software produced output in Excel format. The data from Orthotrak was imported to Matlab 7.0 for analysis. Out of 360 samples, data which completes a gait cycle was taken for analysis i.e. heel strike to heel strike. The data in different planes was analyzed. The CCC between the knee angles was observed for every trial in all the planes. The knee angle was considered because the knee plays a leading role in walking.

\subsection{Data Analysis}

The maximum of CCC was calculated and noted down for each trail. The CCC in the other two planes Frontal and Transversal were also calculated. Figure 2.1 shows the crosscorrelation coefficient profile for walking and walking with mental task. The CCC in these planes varied irregularly and was low too, thus, it was not possible to reach a conclusion. Therefore their values were ig-
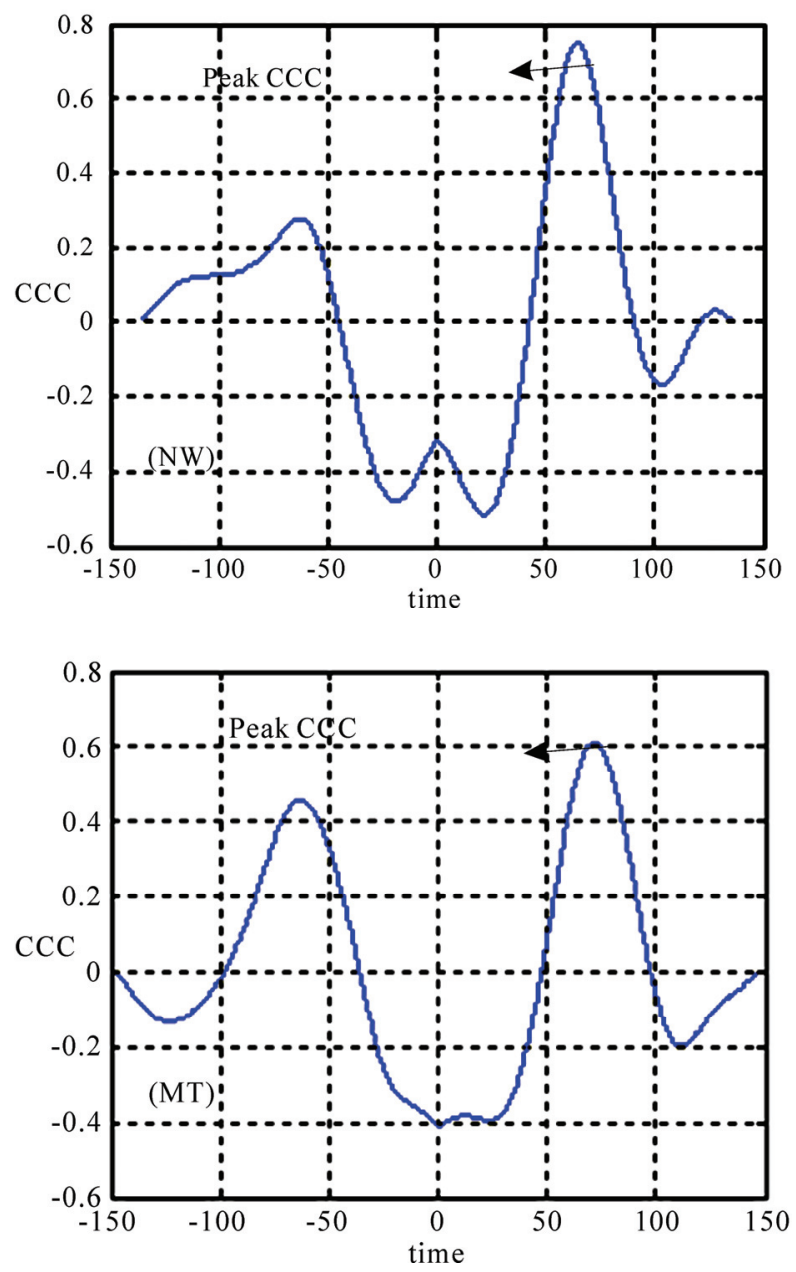

Figure 2 NW denotes Normal Walk and MT denotes walking with mental task. 
nored. The $\mathrm{CCC}$ was calculated using Eq. 1 where $\mathrm{f}$ and $\mathrm{g}$ are left and right knee angle time series, respectively. Cyclogram was drawn as right knee angle vs. left knee angle. Figure 3 shows the cyclogram for while walking and walking with mental task. There was a possibility to synchronize both lower limb gait events, using the lag where peak CCC exists, to draw cyclogram. This was avoided to get any bias of CCC in cyclogram. Cyclogram perimeter is calculated from following Equation:

$$
\mathrm{L}_{\mathrm{i}}=\left\{\left(\varnothing_{\mathrm{lk}}(\mathrm{i}+1)-\emptyset_{\mathrm{lk}}(\mathrm{i})\right)^{2}+\left(\varnothing_{\mathrm{rk}}(\mathrm{i}+1)-\emptyset_{\mathrm{rk}}(\mathrm{i})\right)^{2}\right\}^{1 / 2}
$$

where $\varnothing_{\mathrm{lk}}$ represents the angle of left knee and $\varnothing_{\mathrm{rk}}$ represents the angle of right knee. i represents a particular instant of time. $\mathrm{L}=\sum_{\mathrm{i}=1}^{\mathrm{n}}(\mathrm{Li})$ gives the value of cyclogram perimeter.

The cyclogram perimeter is the total distance travelled in their respective joint spaces. Cyclogram area was not taken into consideration as it is more sensitive to noise comparative to perimeter.
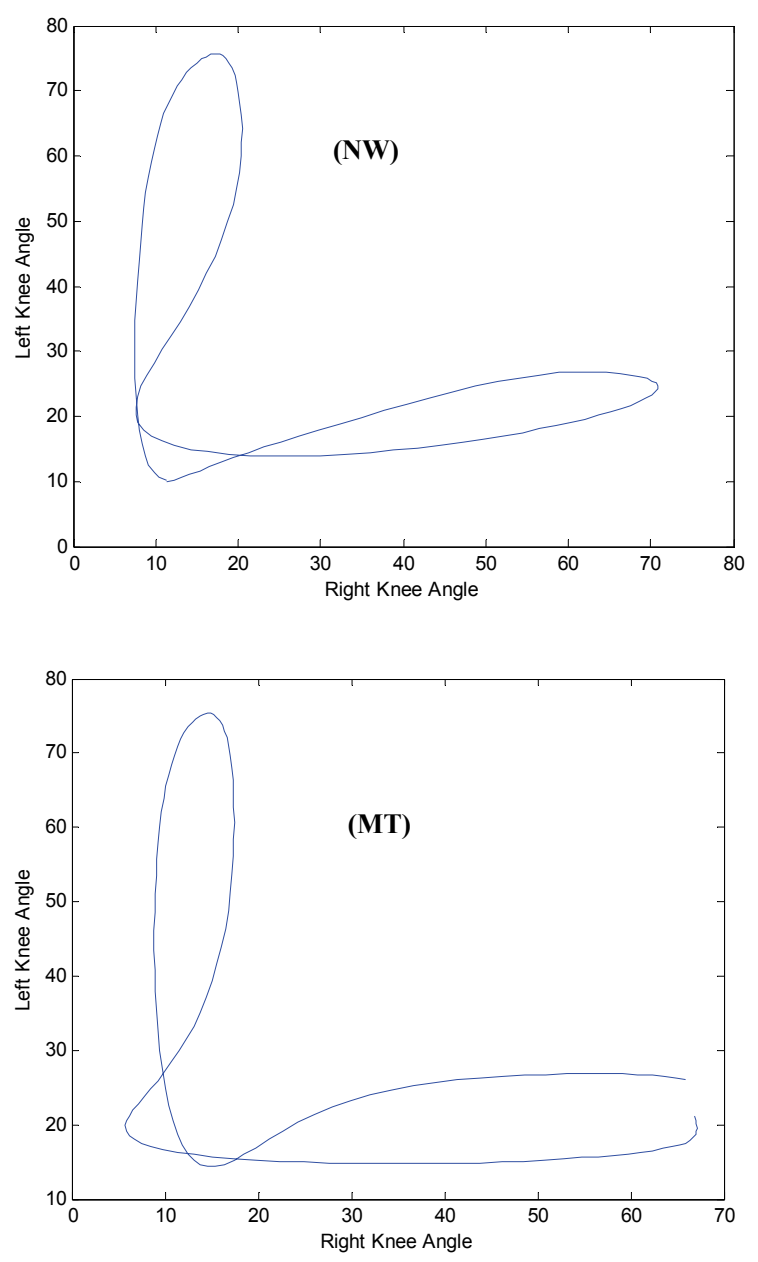

Figure 3 NW denotes Normal Walk and MT denotes walking with mental task.

\section{RESULTS}

\subsection{Crosscorrelation}

The CCC with walking is 0.78 (mean) $\mathrm{SD}=0.03$ which significantly decreases to 0.62 (mean) $\mathrm{SD}=0.01$. The $\mathrm{CCC}$ in the other two planes Frontal and Transversal were also calculated. The CCC in these planes varied irregularly and was low too, thus, it was not possible to reach a conclusion. Therefore their values were ignored.

\subsection{Cyclogram Perimeter}

Cyclogram perimeter decreases with mental task. The normal walk knee-knee cyclogram perimeter matches with the range of hip-knee cyclogram (212.8 degree) calculated at 0 degree slope of walking [13]. The Cyclogram perimeter with walking is 268.2 (mean) $\mathrm{SD}=13.2$ which significantly decreases to 261.9 (mean) $\mathrm{SD}=16.9$.

In contrast to cyclogram perimeter, Crosscorrelation shows higher sensitivity (mean=21.5 S.D. $=2.8$ ) towards the mental task along with gait. The sensitivity calculated for cyclogram perimeter was mean=2.9 S.D. $=1.5$.

\section{DISCUSSION AND CONCLUSIONS}

Methods have been proposed to assess mental task while locomotion. CCC analysis in this research work shows CCC as a good marker to assess mental state. CCC decreases significantly as the walking goes along with the mental task. The results also shows that CCC is highly subjective but it follows the same decreases pattern with mental task. Using Knee angle is more direct measurement in comparision to EMG. Cross correlation and Cyclogram are independent techniques. Though both of them are well established technique to quantify coordination, this study shows CCC is highly sensitive to quantify the change in coordination of limbs, while with mental task. This may be due to the fact that in Crosscorrelation it is the strength of matching of signals to each other with respect to time irrespective of the range the signal (knee angle) covers while in cyclogram it is the sum of range covered by the knee angles trajectories. In terms of implementation in real time in hardware both are equal in terms of complexity. Finally, this study involved a limited number of healthy subjects and the level of education, of subjects, is not taken into account. This work was performed in the $3 \mathrm{D}$ motion analysis lab, a portable, low cost embedded system can be design to calculate CCC and cyclograms of knee angles in the saggittal plane while walking and then can be dedicated for the assesment/toughness of mental task only.

\section{ACKNOWLEDGEMENTS}

The authors highly acknowledge Director, Defence Institute of Physiology and Allied Sciences (DIPAS) New Delhi for allowing the authors to collect data. A sincere acknowledgement to both the subjects for participating voluntarily. 


\section{REFERENCES}

[1] Lord, S. and Rochseter, L. (2007) Walking in real world: Concepts related to functional gait. NZ Journal of Physiotherapy, 35(3).

[2] Satoh, M. and Kuzuhara, S. (2008) Training in mental singing while walking improves gait disturbance in parkinson's patients. Journal of European Neurology, 60(5).

[3] Wannier, T., Bastiaanse, C., Colombo, G. and Dietz, V. (2001) Arm to leg coordination in humans during walking, creeping and swimming activities. Experimental Brain Research, 141, 375-379.

[4] o'Shea, S., Morris, M.E. and Iansek, R. (2002) Dual task interference during gait in people with parkinson disease: Effects of motor versus cognitive secondary tasks. Journal of Physical Therapy, 82( 9).

[5] Patrick, H., Jaket, C., et al. (2000) Interference between gait and coginitive tasks in a rehabilitation neurological application. Journal of Neurol Neurosurg Psychitary, 69, 479-486.

[6] Camicioli, R., Howieson, D., et al. (1997) Talking while walking: The effect of a dual task in aging and Alzheimer's disease. Journal of Neurology, 48(4), 955-958.

[7] Lord, S.E., Rochester, L., et al. (2006) The effect of environment and task on gait parameters after stroke: A randomized comparison of measurement condtions. $\mathrm{Ar}$ chives of Physical Medicine and Rehabilitation, 87(7), 967-973.

[8] Armieri, A., et al. (2009) Dual task performance in a healthy young adult population: Results from a symmetric manipulation of task complexity and articulation. Gait and Posture, 29, 346-348.

[9] Li, L. and Caldwell, G.E. (1999) Coefficient of cross correlation and the time domain correspondence. Journal of Electromyography and Kinesiology, 9, 385-389.

[10] Piek, J.P. (1996) A quantitative analysis of spontaneous kicking in two-month-old infants. Human Movement Science 15, 707-726.

[11] Wren, T.A.L., et al. (2006) Cross-correlation as a method for comparing dynamic electromyography signals during gait. Journal of Biomechanics, 39, 2714-2718.

[12] Haddad, J.M., et al. (2006) Adaptations in interlimb and intralimb coordination to asymmetrical loading in human walking. Gait and Posture, 23, 429-434.

[13] Goswami, A. (1998) A new gait parameterization technique by means of cyclogram moments: Application to human slope walking. Gait and Posture, 8, 15-36.

[14] Jiang,X.Y. and Bunke, H. (1991) Simple and fast computation of moments. Pattern Recognition, 24(8), 801- 806.

[15] Yang, L. and Albregtsen, F. (1996) Fast and exact computation of cartesian geometric moments usig discrete green's theorem. Pattern Recognition, 29(7), 1061-1073.

[16] Charteris, J., Leach, D. and Taves, C. (1979) Comparative kinematic analysis of bipedal and quadrupedal locomotion: A cyclographic technique. Jornal of Analomy, 128(4), 803-819.

[17] Barton, J.G. and Lees, A. (1997) An application of neural networks for distinguishing gait patterns on the basis of hip-knee joint angle diagrams. Gait and posture, 5, 28-33.

[18] Ma, Y.L., Pollick, F.E. and Turner, M. (2005) A statistical approach to gait recognition and verification by using cyclogram. IEEE International Conference on Visual Information Engineering, 425-432.

[19] Hollerbach, J.M., et al. (2001) Torso Force Feedback Realistically Simulates Slope on Treadmill Style Locomotion Interfaces. The International Journal of Robotics Research, 20, 939-951.

[20] Joshi, D., et al. (2009) Gait Co-ordination: Potential marker for mental state. 2nd International Conference in Biomedical Informatics and Signal Processing, 12-14.

[21] Popovic, D. and Jonic, S. (1998) Determining synergy between joint angles during locomotion by radial basis function neural networks. Proceedings of the 20th Annual Conference of the IEEE Engineering on Medicine and Biology Society, 20( 5).

[22] Dejnabadi, H., Jolles, B.M. and Aminian, K. (2008) A new approach for quantitative analysis of inter-Joint coordination during gait. IEEE Transactions on Biomedical Engineering, 55( 2). 